\title{
Lavado de activos y corrupción: análisis desde la perspectiva preventiva como medio para fortalecer la democracia
}

\author{
Money laundering and corruption: analysis from a preventive \\ perspective as a means to strengthen democracy
}

https://doi.org/10.15332/iust.v0i16.2397

\begin{abstract}
Mauricio de la Torre Lascano
Doctor (Ph.D.) por la Universidad de Salamanca (becario), Programa Estado de Derecho y Gobernanza Global. Magister en Gerencia Empresarial MBA, por la Escuela Politécnica Nacional, Diploma Superior en Docencia Universitaria y Auditor por la Universidad Central del Ecuador. Docente titular e investigador en dicha universidad. Becario del gobierno francés para el Ciclo Internacional sobre Administración Pública (CISAP) "Fighting Corruption", Escuela Nacional de Administración (ENA), Francia. Correo electrónico: cdelatorre@uce.edu.ec
\end{abstract}

\begin{abstract}
Resumen
Este trabajo tiene como principal finalidad el análisis de los sistemas para abordar el problema social que genera en los sistemas democráticos el delito de lavado de activos y la corrupción, ya que debilitan las estructuras del Estado. Este estudio inicia con el análisis de doctrina internacional y estudios académicos realizados, tomando en consideración la disyuntiva en adoptar el sistema represivo o preventivo, analizándolos individualmente para identificar cuál sería el más conveniente para fortalecer la democracia en las naciones, considerando que la relación existente entre las medidas de orden preventivo y represivo es inmanente y este flagelo no concluye con la mera represión punitiva, incrementando las penas y sanciones, pero tampoco con un sistema permisivo o laxo. Llegando a determinar como una opción válida la adopción del sistema preventivo en torno a un delito que cada vez se innova con nuevas estratagemas, por lo que se requiere estudios constantes debido a esa especificidad de características particulares que requieren ser analizadas permanentemente.
\end{abstract}

Palabras clave: Corrupción, criminalización, lavado de activos, prevención, políticas públicas.

\begin{abstract}
This paper has as its main purpose the analysis of systems to approach the social problem that generates in the democratic systems the crime of money laundering and corruption, since they weaken the structures of the State. This study begins with the analysis of international doctrine and academic studies carried out, taking into consideration the dilemma in adopting the repressive or preventive system, analyzing them individually to identify which would be the most convenient to strengthen democracy in the nations, considering that existing relationship between the measures of preventive and repressive order are immanent and this scourge does not end only with punitive repression, increasing penalties and sanctions, but neither with a permissive or lax system. Going to determine as a valid option the adoption of the preventive system around a crime that every time innovates with new stratagems, for which constant studies are required due to that specificity of particular characteristics that need to be analyzed permanently.
\end{abstract}

Recibido: 20 de junio de 2018, Aprobado: 15 de octubre de 2018. 
Keywords: Corruption, criminalization, money laundering, prevention, public policy.

\section{Résumé}

Ce travail a pour objectif principal l'analyse de systèmes visant à résoudre le problème social qui engendre dans les systèmes démocratiques le délit de blanchiment d'argent et de corruption, dans la mesure où ils affaiblissent les structures de l'État. Cette étude commence par l'analyse de la doctrine internationale et des études universitaires effectuées, en tenant compte du dilemme posé par l'adoption du système répressif ou préventif, en les analysant individuellement pour déterminer lequel conviendrait le mieux pour renforcer la démocratie dans les nations, étant donné que la relation existante entre les mesures d'ordre préventif et répressif sont immanentes et ce fléau ne s'arrête pas à une simple répression punitive, à des peines plus lourdes et à des sanctions plus lourdes, ni à un système laxiste ou laxiste. En venant de déterminer comme option valable l'adoption du système préventif autour d'un délit qui innove à chaque fois avec de nouveaux stratagèmes, pour lesquels des études constantes sont nécessaires en raison de la spécificité de caractéristiques particulières qui doivent être analysées en permanence.

Mots-clés: Corruption, criminalisation, blanchiment d'argent, prévention, politiques publiques. 


\section{Lavado de activos y corrupción: análisis desde la perspectiva preventiva como medio para fortalecer la democracia $^{1}$}

Mauricio de la Torre Lascano

\section{INTRODUCCIÓN}

“La corrupción es una categoría moral que significa putrefacción y decadencia. Los analistas usan el término para describir aspectos de la vida moderna que consideran repugnantes" (Rose-Ackerman, 2006, p. xiv). La conmoción generalizada en todos los estratos sociales debido a los cada vez más asiduos escándalos a nivel global por hechos de corrupción que responsabilizan a líderes políticos se ha tornado cotidiano, a nivel latinoamericano las investigaciones en los denominados "Panama Papers" y las delaciones en el caso "Lava Jato" involucran a altos funcionarios públicos de elección popular, muchos de los cuales han terminado en prisión, han sido cesados o se encuentran prófugos. Ante lo cual, la sociedad civil se ha organizado y mediante protestas que han sido reprimidas brutalmente con centenares de homicidios hacen conocer su desaprobación a sus gobernantes. "Dichos regímenes no pudieron hacer frente a una combinación de enormes deudas, incremento del desempleo, disturbios urbanos y delincuencia, así como proyectos públicos costosos e ineficientes" (Girling, 2002, p. 28). Los múltiples y variados actos de corrupción cometidos por gobiernos que desean perpetuarse en el poder se están dando a conocer y ya se empiezan a sentir sus devastadores efectos. "La corrupción política es uno de los fenómenos que afecta directa y sustancialmente a la legitimidad y la confianza de las instituciones" (Bautista, 2014, p. 121).

La corrupción no es reciente, de hecho, se señala que esta nace con el ser humano o es tan antigua como la civilización misma, por tanto, su ámbito de aplicación es universal, ya que no disgrega o selecciona una determinada ubicación geográfica, etnia o país. Grupos criminales estructuralmente organizados crean sofisticados esquemas delictivos a gran escala, dejando cuantiosas ganancias provenientes de actividades ilícitas corruptas de diverso orden, entre otras, el narcotráfico, lavado de activos, terrorismo, tráfico y trata de humanos, etc. Delitos que pueden generarse desde el sector público o desde el privado, con especial referencia a los escándalos

1 Artículo científico producto del proyecto de investigación denominado "Lavado de activos: estudio sobre la prevención (en especial referencia al caso ecuatoriano)", desarrollado por el citado autor dentro del programa doctoral Estado de Derecho y Gobernanza Global, bajo la dirección del Dr. Eduardo Fabián Caparrós (Universidad de Salamanca, España). 
mundiales protagonizados por multinacionales, como Enron, Odebrecht, Parmalat o Volkswagen, emergiendo de la sombra de lavado de dinero tras varios casos de alto perfil de las actividades fraudulentas de empresas multinacionales (Ryder, 2011). Es necesario que alguien que haya cometido un hecho delictivo, generando cuantiosos beneficios económicos, se vea obligado a ocultar dicho origen ilegal para evitar ser descubierto y poder disfrutar de los activos (Blanco, 2014), por lo que el lavado de activos o blanqueo de capitales es un mecanismo que las mafias han venido utilizando para legalizar cuantiosas cantidades de dinero, fruto de sus actividades ilícitas, insertando el dinero en la economía de un país, lo que provoca mayor corrupción y socava el Estado de derecho.

La búsqueda de poder no solo se limita dentro de la organización criminal, sino que se expande cada vez más de manera progresiva, considerando la cantidad de flujos económicos que se tratan de esconder e insertar en la economía formal. Fabián, Prado, Blanco \& Zaragoza (2014, p. 41) advierten "los recicladores de capitales ilegales, lejos de colaborar en el progreso del sistema económico, atentan contra el mismo a través del uso anormal que hacen de los instrumentos al servicio de las relaciones de mercado". Y es que los potentes tentáculos de la corrupción en la persecución de hegemonía inclusive financian políticos para poder tener acceso a los sistemas de gobernabilidad. "A nivel de país, leyes electorales deficientes (o la ineficiencia del cumplimiento de buenas leyes) pueden volver muy costosas las elecciones, induciendo a los políticos a buscar fuentes lucrativas para financiar sus campañas" (Banco Mundial, 2009, p. xlix). No obstante, otros efectos secundarios ocurren de manera singular cuando el proceso democrático está desbordado. Un claro ejemplo de ello constituye la concesión a multinacionales extranjeras para explotación de recursos naturales (petróleo, gas, minerales o madera) o adjudicación para la construcción de mega obras, cuyos costos de inversión deben ser compensados por los riesgos implicados, pero se espera que el saldo de sus ganancias generalmente se devengue a la nación propietaria de esos activos. En Estados corruptos, está claro que esto no sucede (Senior, 2006). "El soborno es la forma más común de corrupción pública" (Baker, 2005, p. 50).

La idiosincrasia común en Latinoamérica contribuye a que la corrupción se propague mucho más rápido y de manera generalizada, viéndose muy afectado un factor determinante en las interrelaciones humanas: la confianza, a lo que se suman los comportamientos ordinarios y colectivos de la ciudadanía de omitir la ley con la finalidad de obtener un beneficio personal, alcanzando este fenómeno a todas las funciones del Estado, por tanto la descomposición de las instituciones de la democracia cada vez es mayor. "El rango de confianza en que se mueven los partidos, el Congreso y el poder judicial, las instituciones de la democracia en América Latina en los últimos 20 años no supera los 4 de cada 10 latinoamericanos" (Latinobarómetro, 2015, p. 9); es decir, el nivel de confianza en dichas instituciones es muy pobre, obviamente debido al desencanto permanente de los políticos de turno que permiten que la corrupción siga campeando impunemente. La Organización 
Global de Parlamentarios contra la Corrupción (GOPAC por sus siglas en inglés) señala que "La corrupción es un síntoma de debilidad de los sistemas político, social, jurídico y económico" (GOPAC, 2005, p. 17), y es que no solo las cifras lo demuestran, son los hechos, las acciones que palpamos, percibimos, y nos resignamos en muchos casos a tolerar cotidianamente porque el sistema funciona de esa manera.

En la actualidad, la globalización incide de forma determinante en los Estados, ya que se debe considerar que el uso de transacciones virtuales ha tenido un incremento exponencial, así el desarrollo alcanzado por los mercados financieros provoca sentimientos duales. El avance de las telecomunicaciones, la celeridad en la transferencia de datos, la versatilidad alcanzada por los sistemas de transporte y la homogenización del conocimiento permiten que cada vez se operen mayores volúmenes de dinero en los mercados financieros (Biscayart \& Linares, 2012), flujos económicos que en muchas ocasiones no devienen precisamente de actividades lícitas. La corrupción en las naciones ha engendrado muchos problemas con los que tienen que lidiar los Estados y con ellos riesgos para la ciudadanía que varían considerablemente entre países: extorsión, secuestro y rescate son un problema mucho más grande en muchos países de Europa del Este y América Latina que en la mayoría de los países industrializados, exceptuando partes de Italia (Levi, 2009).

Si bien fue en Norteamérica, en las primeras décadas del siglo pasado, con el tráfico prohibido del alcohol donde se generaban abundantes ganancias (Abel, 2012), con el transcurso del tiempo y el auge del comercio ilegítimo de las sustancias sicotrópicas y estupefacientes, es cuando el lavado de activos empieza a tomar verdadera preponderancia. A partir de los años 70 aumenta la preocupación mundial en torno al reciclaje de dinero, en virtud de la escalada del ingreso de drogas ilegales en los Estados Unidos. Importantísimas sumas de activos son introducidas provenientes del tráfico ilícito de estupefacientes, lo cual determina la decisión de las naciones a luchar contra este delito. De esta manera tuvieron origen poderosas organizaciones transnacionales que pronto extendieron su modalidad delictiva por diversas regiones del mundo (Marengo, 2011), a estos eventos se suma la utilización del sistema financiero global que ya tenía una histórica propensión a la ocultación de sus actividades más comprometidas con su conocido sigilo bancario (Martínez, 2015), que si añadimos la corrupción perpetrada por funcionarios públicos, terminaron fortaleciendo un régimen global de blanqueo de capitales que incluye paraísos fiscales o tax haven.

Se torna necesario, entonces, conocer cuál es la incidencia que tienen el lavado de activos y la corrupción sobre las naciones. Entendiendo que actualmente el desarrollo, tanto de jurisprudencia como de literatura sobre este tema, se ha incrementado y progresado mucho, al punto de incluirlo en las diferentes legislaciones administrativas y penales de los países en forma expresa, especificando qué comprenden estas conductas y qué sanciones conllevan; es decir, tipificándolo, pero tomando en consideración el aporte que brinda el sistema preventivo justamente para fortalecer los sistemas democráticos en las naciones. 
El presente trabajo de investigación se desarrolló con base en un estudio cualitativo exploratorio que abarcó el análisis bibliográfico sobre el delito de lavado de activos y corrupción, tomando en consideración los diferentes sistemas (preventivo y represivo) que existen en torno a la temática, justamente por su naturaleza holística y afectación global en los sistemas democráticos, para lo cual se revisaron varias fuentes documentales, especialmente doctrina, investigaciones y estudios académicos. Aunque existe vasto cúmulo de información sobre la temática, el análisis se ha centrado sobre aquella que tiene correspondencia con la óptica preventiva, ya que ello constituye la base del objetivo central del estudio, que busca demostrar la necesidad de adopción de medidas de orden preventivo para luchar contra este fenómeno que socava las democracias y garantizar que los derechos fundamentales de las sociedades estén protegidos, generando una gobernanza efectiva en las sociedades.

Esta investigación está estructurada de la siguiente manera. Se inicia con un detalle teórico del delito de lavado de activos que incluye definiciones sobre el mismo, llegando a determinar sus múltiples objetivos desde diferentes ópticas. La segunda sección presenta la afectación que genera el lavado de activos y la corrupción en las sociedades, que los convierte en un verdadero problema social. Luego se procede a abordar el problema, tomando en consideración la función que deben cumplir las políticas públicas y la disyuntiva de adoptar el sistema represivo o preventivo, que incluye el tema central de la investigación, haciendo el análisis del problema desde la óptica preventiva. Finalmente, se exponen los resultados que muestran como una opción válida la aplicación del sistema preventivo para fortalecer la democracia.

\section{PERSPECTIVAS TEÓRICAS SOBRE EL DELITO DE LAVADO DE ACTIVOS}

D’Albora (2012) considera que en el caso del lavado de activos su definición jurídica debería ser entendible por el ciudadano común y dentro de esa sencillez, lo define como un proceso complejo, en virtud del cual, bienes de origen delictivo pretenden, en primer término, disimularlo, y luego incorporarse a la economía formal, adquiriendo apariencia de licitud. Hernández (2008) lo determina como el conjunto de mecanismos o procedimientos orientados a dar apariencia de legitimidad o legalidad a bienes o activos de origen delictivo. Lombardero (2009) analiza la finalidad operativa del delito, señalando que el delincuente gozará de los beneficios acumulados con anterioridad, tras legitimar, ocultar la identidad de las ganancias para impedir la detección por las autoridades y permitir que el criminal las use; la integración en el sistema económico legal; la aplicación a actividades económicas lícitas; el incorporar al discurrir de la legitimidad, dar apariencia de legalidad; posibilitar el disfrute jurídicamente incuestionado; traer al tráfico lícito; perder el rastro del origen ilícito del dinero. La actividad criminal para resultar rentable debe ser legalizada en algún momento, de tal manera que sus resultados puedan ser 
disfrutados con tranquilidad. Consecuentemente, el blanqueo de capitales constituye una de las áreas donde más se han enfocado las organizaciones criminales, en la búsqueda de soluciones creativas y en el aprovechamiento de la globalización (Barbero, 2004), ya que este proceso "permite al delincuente el disfrute pacífico de los activos obtenidos por su actividad delictiva previa" (Lombardero, 2009, p. 40). Fabián (1998) lo define como el proceso tendiente a obtener la aplicación en actividades económicas lícitas, de una masa patrimonial derivada de cualquier género de conductas ilícitas, con independencia de cuál sea la forma que esa masa adopte, mediante su progresiva concesión de una apariencia de legalidad, con lo que incluye a organizaciones diferentes de las tradicionales instituciones financieras, estas personas naturales o jurídicas operan en distintos sectores productivos, comerciales o de servicios, ampliándose considerablemente el ámbito donde puede presentarse este delito. Jorge (2008) señala los objetivos básicos del delito de lavado de activos de una manera directa: i. ocultar los delitos que dan origen al dinero, y, ii. asegurar el disfrute de estos ingresos, permitiendo su consumo, inversión o ahorro en la economía legal.

Entre las características más importantes del delito tenemos la globalización de las actividades de blanqueo como consecuencia de su internacionalización, tendencia al profesionalismo y complejidad de nuevos métodos empleados. Todas ellas se encuentran interrelacionadas y se potencian en la medida de su aplicación mundial, la mayor especialización de los blanqueadores da lugar a la elaboración de mecanismos cada vez más sofisticados (Blanco, 2015). La utilización de diferentes y variadas técnicas por parte de los perpetradores son cada vez más complejas, avanzando incluso de manera vanguardista a los entes reguladores, apoyándose en la tecnología, esta posibilidad se incrementa en el caso de la criminalidad económica, ámbito en el que los instrumentos financieros son utilizados para obtener cualquier clase de enriquecimiento ilícito, facilitar la circulación de ese patrimonio ilegal, distanciarlo de su origen delictivo o procurar su reintroducción en el mercado legal (Fabián et al., 2014). Adicionalmente, determina que con la finalidad de legitimar los capitales ilícitos es muy común que se proceda con varios métodos, con lo cual los perpetradores consiguen dos objetivos: a. la acumulación del número de operaciones sobre un mismo patrimonio hace que aumente la distancia que lo separa de su origen, reduciendo las posibilidades que las investigaciones de las autoridades de control permitan establecer un vínculo sólido entre ese capital con su fuente ilícita; b. el fraccionamiento del proceso de blanqueo de un capital en una pluralidad de vías permite diversificar riesgos, garantizando con ello la discreta legalización de un amplio porcentaje del mismo, esquema muy símil a la diversificación de riesgos en el ámbito empresarial con la finalidad de reducir el impacto de unas posibles pérdidas.

El incontenible proceso globalizador en el que se encuentran inmersas las sociedades, así como el desarrollo tecnológico, entre otros factores, han facilitado el crecimiento de forma exponencial de transacciones comerciales, lo que también ha constituido una enorme oportunidad para los grupos delincuenciales para mover, 
transferir u ocultar colosales flujos económicos provenientes de varias y diversas actividades delictivas e insertarlos en la economía formal de las naciones. Gestándose entonces una relación entre el incremento de las ganancias generadas por aquellas actividades criminales con la corrupción de esos flujos financieros y políticas lícitas. Zúñiga (2007) determina que las enormes ganancias generadas por dichas actividades ilícitas, inicialmente generadas por el tráfico de drogas, tienen un poder corruptor sin límites a nivel global, ya que con el establecimiento de la corrupción política y privada se logran dos objetivos claramente identificables: lavado de dinero e impunidad.

\section{LAVADO DE ACTIVOS Y CORRUPCIÓN COMO PROBLEMA SOCIAL}

La corrupción "se refiere al uso de cargos públicos para obtener ganancias personales" (Banco Mundial, 2009, p. xlvi), también se la define como el abuso de una posición pública o privada de confianza para beneficio personal (Ferguson, 2017). "En otras palabras, equivale a violar lo que se considera que está en consonancia con los valores y normas morales pertinentes, igual a la violación de la integridad" (Huberts, 2010, p. 147). La corrupción niega el propósito social positivo: es decir, los medios adecuados para alcanzar un fin valioso (bien común o interés público). Se debe entender que este fenómeno complejo es diferente en las jurisdicciones, dependiendo de diversos factores que facilitan su propagación o diseminación, por lo que hay varios niveles: sistémico, institucional o individual (Gounev \& Bezlov, 2010, p. 28). Transparencia Internacional (2009) clasifica a la "corrupción a gran escala, menor y política, según la cantidad de fondos perdidos y el sector en el que se produzca" (p. 14).

Con total correspondencia, el delito de lavado de activos puede presentarse en cualquier nación, con la particularidad que en aquellos países donde existe menor diversificación de capitales, con controles estatales laxos, normatividad ambigua o excesiva dispersión de esta, se convierten en un campo propicio para que este y otros delitos que devienen de la corrupción se propaguen de manera mucho más agresiva y rápida. Este desarrollo negativo lleva a las naciones inclusive a la denominada corrupción a gran escala, misma que "consiste en actos cometidos en los niveles más altos del gobierno que involucran la distorsión de políticas o de funciones centrales del Estado, y que permiten a los líderes beneficiarse a expensas del bien común" (Transparencia Internacional, 2009, p. 23).

La afectación que genera este delito en las naciones tiene distinto impacto en los denominados países desarrollados y aquellos en vías de desarrollo, en cuyo caso se encuentra la necesidad de esos capitales delictivos para su crecimiento económico, el incremento de producción y consumo de drogas en los países productores e intermediarios, el nivel delincuencial y de marginalidad que hacen del beneficio económico de las actividades delictivas una fuente de riqueza incalculable, además de la corrupción en que están sumidos algunos de esos Estados. Como consecuencia 
se deriva una legislación atrasada, ambigua y laxa en la persecución de delitos, supervisión débil o ineficaz de las actividades financieras, cuerpos de seguridad faltos de formación y motivación para la persecución de delitos y falta de cooperación internacional que posibilite detener las operaciones delictivas originadas en su entorno geográfico (Jiménez, 2009).

El lavado de dinero y la corrupción tienen efectos negativos económicos, sociales y políticos. Los efectos económicos, se refieren a la competencia desleal entre los negocios honestos y deshonestos, distorsión de precios, efecto negativo en la inversión $\mathrm{y}$, eventualmente, exclusión de los negocios honestos. Los efectos sociales incluyen el aumento de corrupción y sobornos. El lavado necesita ayudantes y colaboradores, por lo que cada vez más personas son atraídas hacia la criminalidad. Los efectos políticos devienen en que los delincuentes pueden socavar los sistemas democráticos, convirtiéndolos en altos funcionarios del gobierno de turno (Unger, 2013). Lambsdorff (2006) señala nueve causas que pueden tener incidencia en la corrupción: 1) tamaño del sector público, 2) calidad de la regulación, 3) grado de competencia económica, 4) estructura del gobierno, 5) cantidad de descentralización, 6) impacto de la cultura, 7) valores y el género, y el rol de características invariables, 8) geografía y 9) su historia. Siendo así, es menester que exista en los países una política pública de prevención y lucha contra la corrupción, que armonice en el ámbito nacional lo estipulado en la correspondiente normativa ajustándose a preceptos internacionalmente emitidos. La definición de una política coordinada de control involucrará, antes de iniciar, la toma de conciencia en la entidad obligada acerca de que es parte de un todo en un sistema complejo (Mecikovsky, 2012), abordando el compromiso total en el cumplimiento de las políticas públicas expedidas para que el sistema como tal funcione.

En esta interacción global de elementos o piezas dentro del gran esquema económico mundial que abarca a los Estados, la afectación por la corrupción incide en su desarrollo, fundamentalmente, el lavado de dinero está ligado a la actividad criminal subyacente que lo ha generado. Lavando la actividad criminal que le permite continuar. El lavado de dinero distorsiona los datos económicos y complica los esfuerzos del gobierno para gestionar la política económica (Patel \& Thakkar, 2012), convirtiéndolo en una real amenaza a los Estados que inciden en su desarrollo económico, político, social, jurídico; factores todos negativos que merman el Estado de derecho de las naciones. Havel (2013) determina que "la libertad y la democracia conllevan participación y, por tanto, responsabilidad por parte de todos nosotros" (p. 136). La democracia como la libertad no pueden constituirse como opciones, son verdaderas necesidades.

\section{¿CÓMO ABORDAR EL PROBLEMA?, POLÍTICAS PÚBLICAS Y EL DILEMA ENTRE LA PREVENCIÓN Y REPRESIÓN}

La creación del Estado de derecho abarca la protección de los derechos de propiedad, independencia judicial, incluidos elementos de orden público (Kaufmann, 2004). Determinar las funciones del Estado, órganos, independencia de poderes con 
la finalidad de fiscalizar y juzgar, para buscar la armonía de la sociedad, ha sido una de las tareas clásicas del Derecho administrativo. El Estado fue concebido como una forma de dominación política cuya función principal consistía en garantizar el orden y la paz social. Esta función de garante se ejercía mediante la acción reguladora del Estado (Darnaculleta, 2002).

"El derecho proporciona la necesaria dosis de estabilidad y ello se ve con nitidez en el campo de las instituciones" (Minnicelli, 2013, pp. 17-18), por lo que podemos afirmar que la aplicación de instrumentos legales y formales es necesario para el adecuado desarrollo de las sociedades en el campo jurídico, pero principalmente en el que atañe a la ciudadanía propiamente dicha. La jurisprudencia, la solución a las controversias que se vayan suscitando, constituye un aporte de gran relevancia para la configuración de las reglas y los contenidos del Derecho administrativo, cuyas fórmulas características son las que consiguen un equilibrio entre los poderes administrativos, justificados en la atención de los intereses generales, y los derechos de los particulares (Pardo, 2014), es decir, los Estados se basan en esa interacción de actividades de intereses generales que buscan el equilibrio social. Dentro de este ámbito se construye un sistema ideal de relaciones en el que sus distintos componentes se comportan según pautas y criterios previamente especificados (Oszlak, 1980). Bautista (2017) señala que la teoría política, que estudia las diferentes formas de gobierno, al referirse a la democracia, ofrece acepciones, como: a) proceso de elección de los representantes públicos, b) una forma de gobierno que sirve a la ciudadanía y cumple con los fines de la política, y c) participación de la ciudadanía en las decisiones políticas de su gobierno, lo que se conoce como gobernanza.

El régimen contra el lavado de dinero y la corrupción en esencia se está desarrollando en dos frentes, prevención y represión, y en tres niveles: nacional, regional e internacional (global). La prevención es sobre todo acerca de las sanciones, regulación y supervisión, presentación de informes y medidas de debida diligencia; la represión trata la confiscación, enjuiciamiento y penalización, y las investigaciones, sin embargo, a pesar de la penalización del lavado de dinero y el reciente rol destacado y público de los organismos de represión en el régimen contra este delito, el proceso parece ser sobre todo de tipo reglamentario (Tsingou, 2005). Dos finalidades esenciales se persiguen: i. proteger el sistema financiero y económico, y, ii. colaborar con los tribunales de justicia (Pena, 2013).

La relación existente entre las medidas de orden preventivo y represivo es inmanente, la creación de normativa penal en cada jurisdicción se debe en muchas de las ocasiones por exigencia internacional, el fenómeno de la corrupción no se acaba en la mera represión penal. Solo en la medida que dicha prevención se vincula con medidas de política criminal se puede decir que son medidas accesorias o complementarias al Derecho penal (Etcheberry, 2015).

Lo que se busca con la implementación de políticas públicas, entre otras cosas, es la seguridad como tal, ya sea de orden económico o social, la seguridad es una necesidad en todos los órdenes, si no existe un sustento de paz y orden, ningún plan o 
programa tendrá éxito, pues si no existe orden, tranquilidad y seguridad, no se puede hacer planes, de manera que la seguridad es un presupuesto de casi todo (Herrera, 2002).

"Las políticas públicas corresponden a soluciones específicas de cómo manejar los asuntos públicos" (Lahera, 2004, p. 7), si bien el ámbito en donde se desarrolla la corrupción es tanto público como privado, las normativas correspondientes a identificación, prevención y sanción son emitidas por el Estado. El desarrollo de políticas frente al lavado de activos y los delitos que le preceden introduce complejidades que son inherentes al proceso de regulación estatal de los mercados (Vanoli, 2012), por lo que en función de la realidad y experiencia de cada país se puede optar por cualquiera de los enfoques. La tendencia de los Estados hacia una mayor descentralización ha sido motivada por el desencanto con modos de gobierno centralizados, debido en parte a la percepción de que el gobierno monolítico genera altos niveles de búsqueda de rentas, corrupción y falta de responsabilidad de los funcionarios del gobierno (Bardhan \& Mookherjee, 2006). Las políticas públicas buscan el progreso de las naciones mediante la seguridad, crecimiento y equidad, por lo que se debe tomar en consideración los "entornos políticos y sociales complejos en los que individuos y grupos con un poder de negociación desigual interactúan dentro de reglas cambiantes mientras persiguen intereses en conflicto" (Banco Mundial, 2017, p. 27).

Haciendo una referencia a la innovación permanente del delito de blanqueo Bajo (2009) expone que con la creación del nuevo delito se incurre en dos errores. En primer lugar, creer que las medidas penales son más eficaces que las administrativas o de otro orden en la lucha contra la delincuencia. En segundo lugar, pensar que el Derecho penal es un instrumento ideado para erradicar la delincuencia, basado en la premisa que el Derecho penal es un instrumento que se aplica una vez que los hechos hayan ocurrido.

Resaltando la importancia del sistema represivo, se argumenta que el reto de la justicia penal está en combatir estas prácticas, que tanto daño hacen al Estado, teniendo aquella como meta, no solamente el castigo de los culpables imponiéndoles la pena que corresponda, sino también impedir que aquellos obtengan el beneficio perseguido derivado de las ganancias de su ilícito comportamiento y que la hacienda pública obtenga finalmente el dinero defraudado (Jaén \& Perrino, 2016).

Mientras algunos ordenamientos jurídicos consideran que la adaptación del delito de blanqueo responde a la necesidad de proteger al sistema socioeconómico, otros niegan que exista tal necesidad; considerando que la acción que se quiere incriminar mantiene la lesión al bien jurídico del delito previo. A su vez, una tercera clasificación considera que el delito de blanqueo viene a proteger a la administración de justicia, al igual que lo hace el delito de encubrimiento. Para finalizar, un cuarto grupo de autores y legisladores sostiene que el valor jurídico por proteger resulta ser, fundamentalmente, la seguridad y estabilidad de los Estados, que se ven amenazados por el avance del crimen organizado (Durrieu, 2012). Conclusión emitida, dado el 
nivel de impacto global de afectación del delito de lavado de activos y de la cual se resalta la importancia de la seguridad jurídica como parte fundamental del Estado de derecho.

Se insiste en la afectación de este delito y su incidencia en los niveles de corrupción a nivel general e incluso mundial, ya que el avance de esta en un mundo globalizado refuerza los factores y procesos generales de transnacionalización, contribuye a la desvalorización y pérdida de realidad de las fronteras y soberanías nacionales, amenazando la seguridad nacional (Kaplan, 1996). Lo que interesa destacar es el contexto global de la afectación, aterrizando en un vasto campo que involucra obligatoriamente el esfuerzo común de las naciones, con referencia a la globalización, sus efectos y las iniciativas transnacionales.

\section{Abordaje del problema desde la prevención}

Frente al azote generalizado de todo tipo de actividades ilícitas a nivel global, nace el siguiente cuestionamiento lógico: ¿cómo detenerlas? Prevención refiere a toda una serie de comportamientos y prácticas, tanto individuales como sociales, dirigidas a disminuir la probabilidad de que ciertos eventos dañosos sucedan (Pitch, 2009). Toda estrategia de prevención del delito supone, explícita o implícitamente, la reelaboración de la pregunta sobre qué es el delito, cuáles son sus causas y cómo conjurarlo (Ayos, 2014). En cuanto al marco normativo necesario para la regulación, las leyes contra la delincuencia organizada representan un nuevo paradigma jurídico-dogmático en la conceptualización de delincuencia organizada y en las construcciones jurídicas de los tipos penales, de los instrumentos de prueba y de las reglas de interpretación de pruebas. Existe una razón fáctica: nunca antes organizaciones transnacionales delictivas han sido capaces de atentar contra las sociedades, su democracia, su forma de gobierno y la seguridad de sus ciudadanos como ahora. Su fuerza deriva del mismo proceso de globalización, de las nuevas tecnologías, de la capacidad de adquirir y desarrollar armas de destrucción masiva (Buscaglia, González, Cruz \& Prieto, 2002).

Adicionalmente, las naciones suscriben convenios y en la comunidad mundial se han creado organismos internacionales con la finalidad de prevenir, detectar con oportunidad, sancionar y erradicar estas prácticas criminales. La doctrina internacional determina que existen dos sistemas para combatir este problema: a) preventiva, y b) represiva. Represiva por la ejecución de normas penales, y preventiva mediante la aplicación de normas administrativas, a través del control de ciertas organizaciones, sectores y profesiones. Estos sistemas deben estar intrínsecamente relacionados, contar con retroalimentación y colaboración permanente. Desde los inicios de la coordinación de políticas públicas contra la corrupción, los organismos internacionales optaron por la puesta en práctica de fórmulas de acción coordinada entre el Derecho penal y el Derecho administrativo, basadas en criterios esencialmente cualitativos (Fabián, 2003). En muchas ocasiones existe un conocimiento pleno 
de la actividad delictiva cometida, pero también es muy común que se utilice a terceros para la ejecución, sea como fuere, el delito se realiza y frente al grado de autoría, complicidad u omisión, se condena, ante lo cual Roxin (1981) señala que la culpabilidad es fundamento de la determinación de la pena, de tal forma que el marco para la determinación concreta de la pena se forme por la culpabilidad $\mathrm{y}$ que, dentro de este margen de libertad, sean las consideraciones preventivas las que decidan sobre la magnitud de la pena. La asociación de la pena y el ideal de prevención del delito se sintetiza en tres aspectos: la pena como forma de prevención del delito, en tanto: i. disuasión, ii. corrección y, iii. neutralización (Sozzo, 2014), ya que justamente muchos autores señalan la aplicación de aspectos penales como de ultima ratio.

En referencia al principio de ultima ratio, el proceso de criminalización de una conducta se trata de la respuesta del Estado a los efectos negativos que contiene una conducta humana sobre el individuo y la sociedad en su conjunto, ya que pretender exigir que todo hecho sea subsumido por la ley, es decir, taxatividad absoluta, paralizaría el sistema penal, pues muchos conflictos penales no podrían ser abarcados, lo que terminaría por afectar la función preventiva que está llamado a cumplir (Carnevali, 2008). La penalización de una actuación ocurre entonces, cuando el Estado reprime un tipo de conducta con una pena de contenido punitivo. En consecuencia, cuando dicha conducta es cometida por un individuo, aquel puede ser sometido a un proceso penal y castigado con una sanción de carácter penal (Durrieu, 2012), en el caso del delito de lavado de activos la doctrina estipula que debe ser tipificado como un delito autónomo o independiente, ya que se trata de un crimen pluriofensivo, la evolución de los diferentes instrumentos jurídicos internacionales emitidos permiten ver un progresivo proceso de distanciamiento de la dependencia entre el delito previo y el crimen de blanqueo, dotando a este de progresivos niveles de autonomía (Fabián, 2003). Si bien la corrupción no es para nada reciente, la tipificación propia del acto o de la tentativa de blanquear los productos del delito, sí lo es. Tradicionalmente, la atención penal se centraba sobre el delito que originaba el dinero. La incautación de bienes, en la medida en que se aplicaba por delitos con motivación económica, se configuraba como un castigo contra el delito subyacente. En la actualidad se ha producido un cambio radical (Goite \& Medina, 2015).

El Grupo de Acción Financiera Internacional (GAFI) es considerado como un ejemplo de Derecho administrativo global por la emisión de normativa preventiva, ha conseguido que este delito se integre ya en la mayoría de códigos penales. Pero lo más importante es la regulación para su tratamiento administrativo preventivo (Ballbé, 2007), enfatizando que la eficacia del sistema antiblanqueo puede radicar en la prevención y no solamente en la represión penal. Tradicionalmente en el ámbito del Derecho administrativo sancionador a las personas jurídicas (sociedades, fundaciones, asociaciones, empresas) se las ha venido sancionando muy severamente, sin que la doctrina advirtiera en ello ningún problema, ya que se considera que son 
capaces de realizar infracciones administrativas, puesto que pueden violar de forma culpable las normas que disciplinan su comportamiento (Zugaldía, 2013).

Si bien en un inicio fueron las instituciones financieras las obligadas a reportar información referente al lavado de activos, hoy en día los sistemas actuales de prevención y compliance requieren que una gran gama de organizaciones esté forzada a hacerlo e inclusive sean sancionadas penalmente por omitir información a los entes reguladores. Se sitúa a los sujetos obligados en una posición ciertamente comprometida por la situación de conflicto de intereses que puede aparecer. Se está hablando de empresas con fines comerciales que persiguen maximizar sus beneficios y se presentan ante la sociedad y a sus clientes como instituciones de confianza (Blanco, 2014).

La prevención del delito podría ser la opción más viable, toda vez que el procedimiento preventivo administrativo cumple una función distinta a la normativa penal, su fin no es castigar delitos sino prevenirlos; una normativa preventiva administrativa que regule uniformemente el blanqueo de capitales que proceda del dinero negro y del dinero sucio sería, lo más efectivo para prevenir todos los daños y perjuicios derivados del blanqueo de capitales y de su delito precedente. Es decir, no solo el daño que pueda causar actividades corruptas u otro tipo de delitos, sino el daño que el blanqueo derivado de estos delitos pueda causar al mercado financiero y a todos los entes involucrados en él (García, López \& Mallada, 2015), realzando en el daño en la economía y a la postre en todos los sectores donde la sociedad desarrolla sus actividades, destruyendo el Estado de derecho en las naciones. La importancia de la aplicación mundial o global de normas internacionales y su esquema preventivo, es palpable, ya que todo gira en torno a la instauración de un conjunto de normas de conducta, de carácter obligatorio, que alcanza a un número determinado de actores relevantes del sector político, financiero o la economía en general, los que son expresamente designados como sujetos obligados a prevenir el lavado de activos en el seno de sus esferas de actuación (Blanco, 2011).

Presentando un esquema holístico, resulta útil pensar al régimen jurídico para controlar el lavado de dinero como un sistema estructurado sobre la base de tres pilares fundamentales: por un lado, la prevención y detección, con componentes primordialmente regulatorios, por otro, la investigación penal y el decomiso de bienes $\mathrm{y}$, mediando entre ambos, un híbrido dedicado primordialmente a la investigación financiera, pero que combina algunas funciones de supervisión del cumplimiento regulatorio y otras de colaboración con la investigación penal (Jorge, 2012).

Las medidas dirigidas a impactar directamente en el patrimonio de aquellas personas o entidades que realizan estos graves crímenes y delitos, resultan ser una herramienta fundamental de la moderna política criminal para llevar a cabo este combate; ello es así por cuanto, por un lado, estas acciones permiten cumplir con la máxima de que nadie debe beneficiarse de sus acciones ilícitas; por otro, porque de este modo se logra cortar los circuitos para que sigan financiándose sus empresas criminales; y asimismo, porque el producido de la venta de los bienes primigeniamente 
incautados y luego decomisados, pueden ser aplicados para profundizar la lucha contra estos flagelos y también para ser puestos al servicio del Estado de derecho, para así concretar la función social reparadora que debe asignársele a la recuperación de activos aplicándolos a políticas sociales (Degoumois, 2012), tratándose por tanto de acciones dirigidas a la prevención por el desaliento de ese fin perseguido.

Si el régimen de prevención se limitara a algunos países o no se aplicará de forma homogénea, quienes tratan de lavar el dinero producto de sus delitos lo harían en las jurisdicciones donde su conducta no se encuentra sujeta a controles estrictos (Jorge \& Barreiros, 2012), por tanto es lógico que también existan inconvenientes por diversas causas, incluyendo la aplicación de jurisprudencia y emisión de políticas públicas diseñadas a la medida y lastimosamente también con corte partidista. En ciertas naciones no se han creado aún marcos normativos claros, en línea con la opacidad de su accionar político, es una crítica común que el delito de lavado de dinero en muchas jurisdicciones posee procesos internos innecesariamente complejos, creando un exceso de trámites y pruebas que son muy difíciles de demostrar, lo que disuadirá a los investigadores y fiscales de la focalización de recursos en el delito si ya tienen pruebas suficientes para demostrar el crimen implicado, o quizá simplemente lo ignoran. Especialmente cuando este delito se lleva a cabo en el extranjero y solo el producto de la delincuencia se trata en la jurisdicción (Cheng, 2012).

La labor de prevención debe tener presente que no es a través de la introducción de innumerables y graves sanciones o amenazas de regulación detallada de cada actividad, a través de órganos estatales, como podrá prevenirse la comisión de estos hechos, sino más bien, adoptando todas aquellas medidas que, sin inflar los catálogos punitivos, representen una alternativa de control eficiente y racional, que involucre, además, a las distintas organizaciones interesadas en combatir el fenómeno, con el necesario control estatal, considerando, en primer término, las particularidades de la realidad de cada país (Sánchez \& Montealegre, 2003). Por tal razón, Fabián (1998) deduce que la lucha contra el lavado de capitales debe abordarse preferentemente desde la óptica de la prevención: de lege lata, vigilando la licitud de la causa de las relaciones económicas mediante la atenta aplicación de aquellos instrumentos, específicos o no, con los que se cuente en cada momento; de lege ferenda, previendo la incorporación al ordenamiento jurídico de los mecanismos de control que sean precisos para detectar la realización de operaciones comerciales susceptibles de configurar hipótesis de reciclaje.

El conjunto de características peculiares de cada nación o región, torna más complejo el proceso de estandarización de medidas uniformes. Por lo que resultaría muy conveniente para las naciones que los bienes sociales se distribuyan de acuerdo con las concepciones políticas que prevalecen en una sociedad particular, y no según otras formas de distribución de bienes, como las fuerzas del mercado. "Se entiende que, en una democracia liberal, la política pública debe ser decidida por los ciudadanos, y no por grupos económicos poderosos o quienes poseen riqueza" (Girling, 2002, p. 154). En tanto no se cuente con instancias universales que controlen 
este tipo de procesos, será necesario armonizar las legislaciones de manera que las libertades de la nueva era no perjudiquen sus propios fundamentos (Fabián et al., 2014) y es que la garantía que los derechos fundamentales en general se cumplan en todas las naciones, es decir, que no se vean afectados por los efectos de la corrupción y lavado de activos es una tarea pendiente de aprobación.

\section{CONCLUSIONES}

Existen dos sistemas de abordar el delito de lavado de activos, que como se ha determinado incurre en actividades ilícitas previas, una por la vía represiva, basada en la sanción, en general, mediante la aplicación de los códigos penales, y otra, mediante la utilización de mecanismos disuasivos, haciendo referencia a la emisión de normativa preventiva administrativa. Con lo que, la corrupción, puede derivarse de las condiciones del sistema económico adoptado por las naciones, la política colusoria y las percepciones normativas (sociales). La corrupción no puede evaluarse en términos solamente de una dimensión (económica, política o social). Requiere una síntesis de las tres dimensiones. Una lucha exitosa contra la corrupción, que es realmente una parte del desafío para la gobernanza efectiva de las sociedades, parece requerir una comprensión profunda del vínculo entre las instituciones políticas y la corrupción, considerando la descentralización y accountability (rendición de cuentas) permanente como medidas que apoyarían este proceso.

A nivel mundial se han hecho varias recomendaciones para frenar la corrupción, incluyendo las que ha emitido el GAFI y Transparencia Internacional. Muchos países han incluido dentro de sus códigos penales a la corrupción, estableciendo adicionalmente unidades administrativas especializadas para investigar la corrupción, tales unidades gubernamentales deben estar libres de influencia o injerencia política indebida. En el caso de Ecuador, de manera específica, la corrupción pública no se encuentra tipificada en el Código Orgánico Integral Penal (COIP) de manera explícita o directa a pesar de su reciente emisión y entrada en vigencia en agosto de 2014. Si bien este cuerpo legal prevé el castigo para muchas conductas corruptas como peculado, enriquecimiento ilícito, cohecho, concusión, tráfico de influencias, testaferrismo, entre otras, se ha logrado un paso importante al establecer la responsabilidad penal de las personas jurídicas, aunque hasta el momento no exista jurisprudencia al respecto. La ausencia de tipificación de la corrupción pública de manera explícita constituye un vacío jurídico que permite que muchos sectores políticos, económicos y, como tal, mediante la manipulación de los correspondientes sujetos obligados que operan dentro de ellos, sean utilizados a través de una gama extensa de artilugios para fraguar múltiples delitos, entre ellos el lavado de activos, a pesar de que se encuentre vigente flamante normativa que prohíbe a funcionarios públicos ser propietarios directos o indirectos de bienes o capitales, de cualquier naturaleza, en jurisdicciones o regímenes considerados como paraísos físcales, lo 
que sin duda es un avance jurídico que debe ser robustecido mediante normativa expresa tipificando la corrupción pública.

Dentro del análisis doctrinal referente al proceso del delito, hay que estar claros que existen múltiples y diversas maneras o mecanismos de llevarlo a cabo, utilizando una vasta gama de medios, dependiendo su accionar de esas particularidades propias de cada país, donde la normatividad emitida juega un rol preponderante en la mitigación de efectos y penetración del delito en la sociedad y dentro de ello, a pesar de existir un vasto cúmulo de normativa internacional y doctrina emitida, la adopción del enfoque preventivo o represivo por parte de los Estados busca el equilibrio entre lo benévolo y punitivo en extremo, donde se mantengan las garantías consagradas en los derechos fundamentales, sin perder la hegemonía del sistema es un verdadero reto, toda vez que la falta de entereza política mediante la emisión de normativa de orden preventivo ha producido una propagación del delito a otros sectores económicos en los que operan los sujetos obligados, brindando la oportunidad para que se utilicen nuevos mecanismos, lo que deviene en la adopción con mayor rigurosidad del sistema represivo.

El tratamiento de la corrupción y el delito de lavado de activos desde la perspectiva de la prevención resulta ser una opción válida, toda vez que el objetivo desde este enfoque es garantizar que los derechos fundamentales de las sociedades estén protegidos, por tanto, los efectos y consecuencias negativas que se originan en los sectores económicos donde opera la sociedad en su conjunto se vean reducidos. Los actores en la economía, en referencia a las personas jurídicas e inversores, cuenten con reglas claras, con aportación constante de los involucrados y es aquí donde hace falta mayor interacción, pues como se ha analizado este es un fenómeno que utiliza nuevos mecanismos constantemente, requiriendo especial atención, la aplicación y actualización de instrumentos legales para mitigarlo. El carácter universal del delito examinado y la corrupción requiere además un continuo aporte desde todos los sectores, ya que los esfuerzos realizados nunca serán suficientes para aplacar este mal que azota a las naciones.

\section{REFERENCIAS}

Abel, M. (2012). Blanqueo, innovaciones tecnológicas, amnistía fiscal de 2012 y reforma penal. Revista Electrónica de Ciencia Penal y Criminología, 14(14), 1-45. Recuperado de http://criminet.ugr.es/recpc/14/recpc14-14.pdf

Ayos, E. (2014). Prevención del delito y teorías criminológicas: tres problematizaciones sobre el presente. Estudios Socio-Jurídicos, 16(2), 265-312. Recuperado de http://dx.doi.org/10.12804/esj16.02.2014.09

Bajo, M. (2009). El desatinado delito de blanqueo de capitales. En M. Bajo \& S. Bacigalupo (Eds.). Política criminal y blanqueo de capitales (pp. 11-20). Madrid: Marcial Pons. 
Baker, R. (2005). Capitalism's achilles heel: Dirty money and how to renew the freemarket system. New Jersey: John Wiley \& Sons.

Ballbé, M. (2007). El futuro del Derecho administrativo en la globalización: entre la americanización y la europeaización. Revista de Administración Pública, 174, 215-276. Recuperado de http://www.cepc.gob.es/publicaciones/revistas/revistas electronicas? IDR $=1 \&$ IDN $=626 \&$ IDA $=26537$

Banco Mundial. (2009). Las múltiples caras de la corrupción. Aspectos vulnerables por sectores. Washington, D.C.: Banco Mundial. Recuperado de http:// documents.worldbank.org/curated/en/831401468150568436/pdf/399850PUB0R EPL1f0Corruption1Spanish.pdf

Banco Mundial. (2017). Governance and the Law. Washington, D.C.: Banco Mundial. Recuperado de http://www.worldbank.org/en/publication/wdr2017

Barbero, I. (2004). Delincuencia económica, blanqueo de capitales e inteligencia financiera. Boletín Económico de ICE (2808), 25-34. Recuperado de http:// www.revistasice.com/CachePDF/BICE_2808_25-34_278E74A0035013178FE 69E8A3BA6BEDD.pdf

Bardhan, P. \& Mookherjee, D. (2006). Decentralization, corruption and government accountability. En S. Rose-Ackerman (Ed.). International Handbook on the Economics of Corruption (pp. 161-188). Cheltenham: Edward Elgar.

Bautista, D. (2014). Globalización y corrupción. Previniendo la corrupción en Iberoamérica mediante la ética pública en los albores del siglo XXI. Dilemata, 6(15), 118-129. Recuperado de http://www.dilemata.net/revista/index.php/ dilemata/article/viewFile/295/315

Bautista, D. (2017). De la democracia corrupta a la democracia ética. Sugerencias para la consolidación de la democracia en México. En D. Bautista \& L. Rodríguez (Coords.). Madurez ciudadana. Requisito para la consolidación de una democracia ética (pp. 21-41). Toluca: Centro de Investigación en Ciencias Sociales y Humanidades.

Biscayart, J. \& Linares, M. (2012). Lavado de dinero: Responsabilidad judicial en el marco de los compromisos internacionales asumidos en la materia. En H. Bertazza y F. D’Albora (Dirs.). Tratado de lavado de activos y financiación del terrorismo: Prevención, investigación y represión, tomo I (pp. 29-42). Buenos Aires: La Ley.

Blanco, I. (2014). La prevención del blanqueo de capitales. En Tirant Lo Blanch (Ed.). Manual de cumplimiento normativo y responsabilidad penal de las personas jurídicas (pp. 439-498). Valencia: Tirant Lo Blanch.

Blanco, I. (2015). El delito de blanqueo de capitales. Navarra: Aranzadi.

Blanco, H. (2011). Lavado de activos por sujetos obligados. Análisis desde la teoría de los delitos de infracción de deber. Buenos Aires: Abeledo-Perrot. 
Buscaglia, E., González, S., Cruz, J. \& Prieto, C. (2002). Corrupción y delincuencia organizada. Un estrecho vínculo. Revista Universitaria (76), 55-62. Recuperado de https://www.unifr.ch/ddp1/derechopenal/obrasportales/op_20080612_03.pdf

Carnevali, R. (2008). Derecho penal como ultima ratio. Hacia una política criminal racional. Ius et Praxis, 14(1), 13-48. https://dx.doi.org/10.4067/S071800122008000100002

Cheng, N. (2012). The Effectiveness of Money Laundering Investigations in Fighting Transnational Crime: A Comparison of the United States and Hong Kong. Washington D.C.: The Brookings Institution. Recuperado de https://www. brookings.edu/wp-content/uploads/2016/06/03_money_laundering_cheng.pdf

Darnaculleta, M. (2002). Derecho administrativo y autorregulación: la autorregulación regulada. (Tesis inédita de doctorado en Derecho Público). Universitat de Girona, España. Recuperado de http://www.tdx.cat/ handle/10803/7681

D’Albora, F. (2012). La prevención y el control del lavado de dinero. En H. Bertazza \& F. D’Albora (Dirs.). Tratado de lavado de activos y financiación del terrorismo: Prevención, investigación y represión, tomo I (pp. 177-205). Buenos Aires: La Ley.

Degoumois, M. (2012). Monitoreo de la implementación de políticas contra el lavado de activos y el financiamiento del terrorismo. En D. Sarrabayrouse \& M. Degoumois (Coords.). Lavado de activos: prevención y sanción (pp. 89-104). Buenos Aires: Infojus.

Durrieu, R. (2012). Hacia un delito autónomo y pluriofensivo de lavado de dinero. En H. Bertazza y F. D’Albora (Dirs.). Tratado de lavado de activos y financiación del terrorismo: Prevención, investigación y represión, tomo I (pp. 153-174). Buenos Aires: La Ley.

Etcheberry, J. (2015). La regulación internacional del lavado de activos y el financiamiento del terrorismo. En K. Ambos, C. Caro \& E. Malarino (Coords.). Lavado de activos y compliance. Perspectiva internacional y derecho comparado (pp. 91-142). Lima: Jurista Editores.

Fabián, E., Prado, V., Blanco, I. \& Zaragoza, J. (2014). Combate al Lavado de Activos desde el Sistema Judicial. Edición Especial para el Perú. Washington, DC: CICAD.

Fabián, E. (1998). El delito de blanqueo de capitales. Madrid: Editorial Colex.

Fabián, E. (2003). El abogado frente al blanqueo de capitales. Revista del INACIPE Iter Criminis (5), 1-35.

Ferguson, G. (2017). Global Corruption: Law, Theory and Practice. Washington, DC: UNODC. Recuperado de https://track.unodc.org/Academia/Pages/ TeachingMaterials/GlobalCorruptionBook.aspx

García, C., López, F. \& Mallada, C. (2015). Guía práctica de prevención del blanqueo de capitales. Pamplona: Aranzadi. 
Girling, J. (2002). Corruption, Capitalism and Democracy. Londres: Routledge.

Goite, M. \& Medina, A. (2015). El delito de lavado de activos: necesario análisis desde una dimensión plural. En G. Armienta, M. Goyte, A. Medina. L. Gambino \& L. García (Coords.). El lavado de dinero en el siglo XXI. Una visión desde los instrumentos jurídicos internacionales, la doctrina y las leyes en América Latina y España (pp. 21-66). Sinaloa: Unijuris.

Gounev, P. \& Bezlov, T. (2010). Examining the links between organised crime and corruption. Sofia: Center for the Study of Democracy.

Havel, V. (2013). El poder de los sin poder. Madrid: Encuentro.

Hernández, R. (2008). Prevención del blanqueo de capitales: respuestas a las dudas de los profesionales jurídicos. Diario La Ley, año XXIX, no. 7020, 25 septiembre 2008, (pp. 1-3). Recuperado de http://diariolaley.laley.es/content/Inicio.aspx

Herrera, A. (2002). La prevención de los delitos: elemento fundamental en la seguridad pública. Revista de Administración Pública (106), 73-91. Recuperado de www.juridicas.unam.mx/publica/librev/rev/rap/cont/106/pr/pr6.pdf

Huberts, L. (2010). A Multi Approach in Corruption Research: Towards a More Comprehensive Multi-Level Framework to Study Corruption and Its Causes. En G. de Graaf, P. von Maravic \& P. Wagenaar (Eds.). The Good Cause. Theoretical Perspectives on Corruption (pp. 146-165). Leverkusen: Barbara Budrich Publishers.

Jaén, M. \& Perrino, A. (2016). La recuperación de activos frente a la corrupción. La Oficina de Recuperación y Gestión de Activos. Madrid: Dykinson.

Jiménez, C. (2009). Blanqueo de capitales. (Tesis inédita de doctorado en Derecho Privado). Universidad Rey Juan Carlos, España. Recuperado de http://docplayer. es/2023550-El-blanqueo-de-capitales.html

Jorge, G. (2008). Recuperación activos de la corrupción. Buenos Aires: Del Puerto. Jorge, G. (2012). Políticas de control del lavado de dinero. En H. Bertazza y F. D'Albora (Dirs.). Tratado de lavado de activos y financiación del terrorismo: Prevención, investigación y represión, tomo I (pp. 43-104). Buenos Aires: La Ley.

Jorge, G. \& Barreiros, L. (2012). Estándares internacionales en materia de prevención del lavado de dinero. En H. Bertazza y F. D’Albora (Dirs.). Tratado de lavado de activos y financiación del terrorismo: Prevención, investigación y represión, tomo I (pp. 105-145). Buenos Aires: La Ley.

Kaplan, M. (1996). Economía criminal y lavado de activos. Boletín Mexicano de Derecho Comparado, 1(85), 217-241. Recuperado de http://revistas.juridicas. unam.mx/index.php/derecho-comparado/article/view/3410/3982

Kaufmann, D. (2004). Governance Redux: The Empirical Challenge. Washington D.C.: World Bank Institute. Recuperado de http://siteresources.worldbank.org/ INTWBIGOVANTCOR/Resources/govredux.pdf 
Lahera, E. (2004). Introducción a las Políticas Públicas. Santiago de Chile: Fondo de Cultura Económica.

Lambsdorff, J. (2006). Causes and consequences of corruption: What do we know from a cross-section of countries? En S. Rose-Ackerman (Ed.). International Handbook on the Economics of Corruption (pp. 3-51). Cheltenham: Edward Elgar.

Latinobarómetro. (2015). La confianza en América Latina 1995 - 2015. 20 años de opinión pública latinoamericana. Santiago de Chile: Latinobarómetro. Recuperado de http://www.latinobarometro.org/latNewsShow.jsp

Levi, M. (2009). White-Collar Crimes and the Fear of Crime: A Review. En S. Simpson \& D. Weisburd (Eds.). The Criminology of White-Collar Crime (pp. 79-109). New York: Springer.

Lombardero, L. (2009). Blanqueo de capitales. Prevención y represión del fenómeno desde la perspectiva penal, mercantil, administrativa y tributaria. Barcelona: Bosch.

Marengo, F. (2011). Aspectos generales del lavado de activos. Revista Pensamiento Penal, 1-20. Recuperado de http://www.pensamientopenal.com.ar/system/ files/2014/12/doctrina28927.pdf

Martínez, J. (2015). Estrategias multidisciplinarias de seguridad para prevenir el crimen organizado. (Tesis inédita de doctorado en Derecho Público). Universidad de Barcelona, España. Recuperado de http://hdl.handle.net/10803/298308

Mecikovsky, J. (2012). Manual de procedimientos para la prevención del lavado de dinero y financiamiento del terrorismo. En H. Bertazza y F. D'Albora (Dirs.). Tratado de lavado de activos y financiación del terrorismo: Prevención, investigación y represión, tomo III (pp. 163-199). Buenos Aires: La Ley.

Minnicelli, A. (2013). Eficacia. Ideas y tensiones para la articulación actual de políticas públicas y derecho administrativo. Buenos Aires: Atlántida.

Organización Global de Parlamentarios contra la Corrupción. (2005). Control de la corrupción: Manual del parlamentario. Ottawa: GOPAC. Recuperado de http:// gopacnetwork.org/Docs/CCH_Final_ES.pdf

Oszlak, O. (1980). Políticas públicas y regímenes políticos: Reflexiones a partir de algunas experiencias latinoamericanas. Estudios CEDES, 3(2), 1-38. Recuperado de http://www.cedes.org.ar/Publicaciones/Est_c/Est_c3,2.pdf

Pardo, J. (2014). Lecciones de derecho administrativo. (4⿳亠丷⿵冂丶 ${ }^{\mathrm{a}}$ ed.). Madrid: Marcial Pons.

Patel, H. \& Thakkar, B. (2012). Money Laundering Among Globalized World. En H. Cuadra-Montiel (Ed.). Globalization - Approaches to Diversity (pp. 163-182). Rijeka: InTechOpen.

Pena, A. (2013). Manual de prevención del blanqueo de capitales para profesionales. Santiago de Compostela: Andavira Editora.

Pitch, T. (2009). La sociedad de la prevención. Buenos Aires: Ad-Hoc. 
Rose-Ackerman, S. (2006). Introduction and overview. En S. Rose-Ackerman (Ed.). International Handbook on the Economics of Corruption (pp. xiv-xxxviii). Cheltenham: Edward Elgar.

Roxin, C. (1981). Culpabilidad y prevención en derecho penal. Madrid: Editorial Reus.

Ryder, N. (2011). Financial crime in the 21st century. Northampton: Edward Elgar Publishing Limited.

Sánchez, C. \& Montealegre, F. (2003). Lavado de dinero y activos provenientes de actividades ilícitas aspectos doctrinarios y normativos. Revista de Derecho (4), 145-185. Recuperado de http://www.revistasnicaragua.net.ni/index.php/ revderecho/article/view/944

Senior, I. (2006). Corruption - the World's Big C. Cases, Causes, Consequences, Cures. Londres: The Institute of Economic Affairs.

Sozzo, M. (2014). Prevención del delito. Estrategias, efectos y dilemas. Quito: FGE.

Transparencia Internacional. (2009). Guía de lenguaje claro sobre lucha contra la corrupción. Berlín: Transparencia Internacional. Recuperado de http://www. transparency.org/whatwedo/publication/guia_de_lenguaje_claro_sobre_lucha_ contra_la_corrupcion

Tsingou, E. (2005). Global governance and transnational financial crime opportunities and tensions in the global anti- money laundering regime. Centre for the Study of Globalisation and Regionalisation, Working paper 161/05, 1-25. Recuperado de http://wrap.warwick.ac.uk/1959/1/WRAP_Tsingou_wp16105.pdf

Unger, B. (2013). "Introduction”. En B. Unger y D. Van der Linde (Eds.). Research Handbook on Money Laundering (pp. 3-18). Cheltenham: Edwar Elgar.

Vanoli, A. (2012). El rol de la CNV en la prevención y persecución del lavado de activos a través del mercado de capitales. En D. Sarrabayrouse \& M. Degoumois (Coords.). Lavado de activos: prevención y sanción (pp. 115-127). Buenos Aires: Infojus.

Zugaldía, J. (2013). La responsabilidad criminal de las personas jurídicas, de los entes sin personalidad y de sus directivos. Valencia: Tirant Lo Blanch.

Zúñiga, L. (2007). Criminalidad organizada, Derecho penal y sociedad. Apuntes para el análisis. En Contribuciones críticas al sistema penal de la post modernidad, in memoriam a Eduardo Novoa Monreal (pp. 173-195). Santiago de Chile: Universidad Central de Chile. 